Received by the editors: October 03, 2019; Accepted: July 20, 2020

\title{
ANNIHILATORS OF POWER VALUES OF $b$-GENERALIZED DERIVATIONS IN PRIME RINGS
}

\author{
Nihan Baydar YARBİL \\ Department of Mathematics Faculty of Science Ege University, İzmir, TURKEY
}

\begin{abstract}
Let $R$ be a prime ring with extended centroid $C$ and maximal left ring of quotients $Q_{m l}(R)$. For a nonzero element $b \in R$, let $F: R \rightarrow R$ be a right $b$-generalized derivation associated with the map $d$ of $R$. Suppose that $s(F(x))^{n}=0$ for all $x \in R$ where $s$ is a nonzero element in $R$ and $n \geq 1$ is a fixed positive integer. Then there exist some $c \in Q_{m l}(R)$ and $\beta \in C$ such that $d(x)=a d_{c}(x), F(x)=(c+\beta) x b$ for all $x \in R$ and either $s(c+\beta)=0$ or $b(c+\beta)=0$.
\end{abstract}

\section{Introduction}

Throughout this paper $R$ will always denote a prime ring with center $Z(R)$, extended centroid $C$, left maximal ring of quotients (respectively right maximal ring of quotients) $Q_{m l}(R)$ (resp. $Q_{m r}(R)$ ), the Martindale ring of quotients $Q(R)$ and the symmetric Martindale quotient ring $Q_{s}(R)$. In case $R$ is a prime ring, $Q(R), Q_{m l}(R), Q_{m r}(R), Q_{s}(R)$ are also a prime rings and $C$ is a field. For more details the book [1] is referred.

By a derivation of $R$ we mean an additive map $d$ of $R$ satisfying $d(x y)=d(x) y+$ $x d(y)$ for all $x, y \in R$. For $a \in R$, the map $d: R \rightarrow R$ defined by $d(x)=[a, x]$ for all $x \in R$ is called an $X$-inner derivation and denoted by $d(x)=a d_{a}(x)$. It is well known that any derivation $d$ of $R$ can be uniquely extended to $Q_{m l}(R)$ (or $Q_{m r}(R)$ ). Hence any extension of an $X$-inner derivation is also $X$-inner which can be induced by an element $q \in Q_{s}(R)$, i.e., $d(x)=a d_{q}(x)$. A generalized derivation $g$ is an additive map of $R$ satisfying $g(x y)=g(x) y+x d(y)$ for all $x, y \in R$ where $d$ is a derivation of $R$ which is uniquely determined by $g$ and is called the associated derivation of $g$.

2020 Mathematics Subject Classification. Primary 16N60 ; Secondary 16W25.

Keywords and phrases. Prime ring, generalized polynomial identity,b-generalized derivation.

nihan.baydar.yarbil@ege.edu.tr

(D) 0000-0003-1376-2349.

(C) 2020 Ankara University Communications Faculty of Sciences University of Ankara-Series A1 Mathematics and Statistics 
Let $\alpha, \beta$ be automorphisms of $R$. An $(\alpha, \beta)$-derivation of $R$ is an additive mapping $\delta: R \rightarrow R$ such that $\delta(x y)=\delta(x) \alpha(y)+\beta(x) \delta(y)$ for all $x, y \in R$. If $\alpha=I d$, the identity automorphism of $R$, then $\delta$ is called a $\beta$-derivation or simply called a skew derivation. An additive map $\delta$ of $R$ is a generalized $\alpha$-derivation if there exists an $\alpha$-derivation $d$ of $R$ such that $\delta(x y)=\delta(x) y+\alpha(x) d(y)$ for all $x, y \in R$.

The notion of $b$-generalized derivations is introduced by Koşan and Lee in 10]. They define generalized $b$-derivations as in the following:

(1) Let $d: R \rightarrow Q_{m r}(R)$ be an additive map. An additive map $F: R \rightarrow$ $Q_{m r}(R)$ is called a left b-generalized derivation with the associated map $d$ if $F(x y)=F(x) y+b x d(y)$ for all $x, y \in R$.

(2) Let $d: R \rightarrow Q_{m l}(R)$ be an additive map. An additive map $F: R \rightarrow$ $Q_{m l}(R)$ is called a right b-generalized derivation with the associated map $d$ if $F(x y)=x F(y)+x d(y) b$ for all $x, y \in R$.

When treating an additive map of a ring $R$, the main goal is to give a characterization of the map or to state some structural results related to the ring itself. The works have been done related to power values of some kind of additive maps so far was initiated in 8. Giambruno and Herstein proved that if $R$ is a semiprime ring and $d$ is a derivation of $R$ such that $d(x)^{n}=0$ for all $x \in R$, where $n \geq 1$ is a fixed integer, then $d=0$. The generalization of this problem has been worked by a number of researchers. For instance in 2 Brešar proved that if $R$ is a prime ring with a nonzero derivation $d$ and $a$ is an element of $R$ such that $a d(x)^{n}=0$ for all $x \in R$, where $n \geq 1$ is a fixed positive integer, then $a=0$ in case $\operatorname{char} R \neq(n-1)$ !. In [11 Lee and Lin obtained the same result on some noncentral Lie ideal of $R$ without the assumption on the characteristic.

In [3] J.C. Chang proved that if $R$ is a prime ring and $\delta$ is a right generalized $(\alpha, \beta)$-derivation of $R$ such that $a \delta(x)^{n}=0$ for all $x \in R$ and some $a \in R$ where $n \geq 1$ is a positive integer, then $\delta=0$ or $a=0$.

Motivated by these results we prove the following theorem.

Theorem 1. Let $R$ be a prime ring, $F$ be a nonzero right b-generalized derivation of $R$ associated with the map $d$ of $R$ and $s, b$ be nonzero elements in $R$. If $s(F(x))^{n}=0$ for all $x \in R$ where $n$ is a fixed positive integer, then there exist $c \in Q_{m l}$ and $\beta \in C$ such that $d(x)=a d_{c}(x), F(x)=(c+\beta) x b$ and either $s(c+\beta)=0$ or $b(c+\beta)=0$.

\section{Preliminaries}

It is well known that the automorphisms, derivations, generalized derivations and $\alpha$-derivations of $R$ can be uniquely extended to $Q(R), Q_{m r}(R)$ and $Q_{m l}(R)$. For sure the results obtained for $Q_{m r}(R)$ can be adapted to $Q_{m l}(R)$ as well.

Before presenting the results we will state the following remarks:

Fact 1. Let $T=Q_{m r}(R) *_{C} C\left\{X_{1}, X_{2}, \ldots\right\}$ be the free product of $C$-algebras $Q_{m r}(R)$ and $C\left\{X_{1}, X_{2}, \ldots\right\}$ where $C\left\{X_{1}, X_{2}, \ldots\right\}$ is the free algebra in noncommutative indeterminates $\left\{X_{1}, \ldots, X_{2}\right\}$ over $C$. Let $f\left(X_{i}\right) \in T$, then $f$ is called a GPI 
(that is, a generalized polynomial identity) of $T$ if $f\left(x_{i}\right)=0$ for all $x_{i} \in T$.

Fact 2. Let $R$ be a prime ring and $I$ be a two-sided ideal of $R$. Then $I, R$ and $Q_{m r}(R)$ satisfy the same generalized polynomial identities with coefficients in $Q_{m r}(R)$ (see [4]).

Fact 3. (Lemma 2 in [4]) Let $R$ be a prime ring and $Q_{m r}(R)$ is the maximal right ring of quotients of $R$. Suppose that $B$ is a basis of $Q_{m r}(R)$ over $C$. Let $\alpha_{i}, q_{i} \in B$, then $m_{i}=q_{0} y_{1} q_{1} \ldots y_{n} q_{n}$ are called monomials and hence any element in the free product $T$ is of the form $g=\sum_{i} \alpha_{i} m_{i}$. In that case, the generalized polynomial $g=\sum_{i} \alpha_{i} m_{i}$ is zero in $T$ if and only if $\alpha_{i}=0$ for all $i$. Consequently for $h_{i}\left(X_{1}, \ldots, X_{n}\right), k_{i}\left(X_{1}, \ldots, X_{n}\right) \in T$ and

$$
\begin{aligned}
& g_{1}\left(X_{1}, \ldots, X_{n}\right)=\sum_{i=1}^{n} X_{i} h_{i}\left(X_{1}, \ldots, X_{n}\right), \\
& g_{2}\left(X_{1}, \ldots, X_{n}\right)=\sum_{i=1}^{n} X_{i} k_{i}\left(X_{1}, \ldots, X_{n}\right)
\end{aligned}
$$

if $a_{1}, a_{2} \in Q_{m r}(R)$ are linearly independent over $C$ and

$$
a_{1} g_{1}\left(X_{1}, \ldots, X_{n}\right)+a_{2} g_{2}\left(X_{1}, \ldots, X_{n}\right)=0 \in T,
$$

then both $g_{1}\left(X_{1}, \ldots, X_{n}\right)$ and $g_{2}\left(X_{1}, \ldots, X_{n}\right)$ are zero in $T$.

In the present paper, we consider $F$ as a right $b$-generalized derivation of $R$ with associated map $d$ of $R$ and $b \in R$. It is proved in $[10$ that if $R$ is a prime ring with $b \neq 0$, then the associated map $d$ must be a derivation of $R$. For some $a, b, c \in Q_{m l}(R)$, the map $F(x)=a x+c x b$ is an example of right $b$-generalized derivation of $R$ and called $X$-inner right b-generalized derivation.

\section{The CASE OF INNER $b$-GeneraLized DERIVATIONS}

Lemma 2. Let $R$ be a prime ring and $s, a, b, c \in R$ with $b, s \neq 0$. Suppose that $s(x a+c x b)^{n}=0$ for all $x \in R$ where $n$ is a fixed positive integer. If $R$ does not satisfy any nontrivial generalized polynomial identity, then there exists some $\beta \in C$ such that $a=\beta b$ and either $s(c+\beta)=0$ or $b(c+\beta)=0$.

Proof. If $R$ does not satisfy any nontrivial generalized polynomial identity, then

$$
s(X a+c X b)^{n}=0 \in T .
$$

We suppose that $a \neq 0$. By (1),

$$
s(X a+c X b)^{n-1} X a+s(X a+c X b)^{n-1} c X b=0 \in T .
$$

First we consider the case when $a$ and $b$ are linearly $C$-independent. Then using Fact 3, we have $s(X a+c X b)^{n-1} X a=0$. The monomial $s(X a)^{n}$ is nontrivial in the last equation since $s \neq 0$ and $a \neq 0$, which contradicts with the assumption that $R$ 
is not a GPI-ring. Thus $a$ and $b$ must be linearly dependent on $C$. In that case, there exists some $\beta \in C$ such that $a=\beta b$. Writing this in (1), we have

$$
0=s(X \beta b+c X b)^{n}=s(c+\beta)(X b(c+\beta))^{n} .
$$

In view of Theorem 2 in $[7$, we obtain $s(c+\beta)=0$ or $b(c+\beta)=0$.

Lemma 3. Let $R$ be a prime ring $s, a, b, c \in R$ with $s, b \neq 0$. Suppose that $s((x a+c x) b)^{n}=0$ for all $x \in R$ where $n$ is a fixed positive integer. Then there exists $\beta \in C$ such that $(a-\beta) b=0$ and either $s(c+\beta)=0$ or $b(c+\beta)=0$.

Proof. First suppose that $R$ does not satisfy any nontrivial generalized polynomial identity. Then

$$
s((X a+c X) b)^{n}=0 \in T .
$$

Suppose that $s \neq 0, a \neq 0$. By $(2)$ we have

$$
s((X a+c X) b)^{n-1} X a b+((X a+c X) b)^{n-1} c X b=0 \in T .
$$

Now consider the case when $a b$ and $b$ are linearly $C$-independent. Using Fact 3 we obtain $s((X a+c X) b)^{n-1} X a b=0 \in T$. The monomial $s(X a b)^{n}$ is nontrivial since $s \neq 0, a \neq 0$, which contradicts with the assumption on $R$ for not being a GPI-ring. Hence $a b$ and $b$ must be linearly dependent over $C$. In that case, there exists some $\beta \in C$ such that $(a-\beta) b=0$. So the equation 2 becomes

$$
\begin{aligned}
0 & =s((X a+c X) b)^{n} \\
& =s((X(a-\beta)+(c+\beta) X) b)^{n} \\
& =s(c+\beta)(X b(c+\beta))^{n} .
\end{aligned}
$$

In view of Theorem 2 in $[7$, we have $s(c+\beta)=0$ or $b(c+\beta)=0$.

Now suppose that $R$ satisfies a nontrivial generalized polynomial identity, i.e., $R$ is a GPI-ring. By Theorem 2 in $[4$

$$
s((x a+c x) b)^{n}=0
$$

for all $x \in R C$. If $C$ is an infinite field, then let $F$ denote the algebraic closure of $C$ and if $C$ is a finite field, then let $F=C$. Hence the equation (4) holds for all $x \in R^{\prime}$ where $R^{\prime}=R C \otimes_{C} F$. In the light of Theorem 3.5 in [5], $R^{\prime}$ is a centrally closed prime $F$-algebra. By Theorem 3 in [13], $R^{\prime}$ is a primitive ring with a minimal idempotent $e$ such that $e R^{\prime} e=F e$ and there exists a right vector space $V_{F}$ such that $R^{\prime}$ acts densely on $V_{F}$.

For a given $v \in V$, the first aim is to see that $(a b) v$ and $b v$ are linearly dependent over $F$. First suppose that $(a b) v$ and $b v$ are linearly independent over $F$. By the density of $R^{\prime}$, there exists $x \in R^{\prime}$ such that $x(a b) v=v, x b v=0$. Using this in (4), we obtain

$$
0=s((x a+c x) b)^{n} v=s v .
$$

Since the action of $R^{\prime}$ on $V_{F}$ is faithful then $s=0$, which leads a contradiction. Thus we may assume that $a(b v)$ and $b v$ are linearly dependent on $F$. 
Case 1. If $\operatorname{dim}_{F}(b V)=1$, then we may choose $v^{\prime} \in V$ such that $b V=b v^{\prime} F$ and write $a b v^{\prime}=b v^{\prime} \gamma$ for some $\gamma \in F$. Let $v \in V$ and $b v=\alpha^{\prime} b v^{\prime}$ for some $\alpha^{\prime} \in F$. Then

$$
a b v=a \alpha^{\prime} b v^{\prime}=\alpha^{\prime} a b v^{\prime}=\gamma^{\prime} \alpha^{\prime} b v^{\prime}=\gamma^{\prime} b v .
$$

that is $a b v$ and $b v$ are linearly $F$-dependent. The equation

$$
a b v=\gamma^{\prime} b v
$$

gives $a b=\gamma^{\prime} b$ since the action of $R^{\prime}$ on $V_{F}$ is faithful.

Case 2. If $\operatorname{dim}_{F}(b V) \geq 2$, then there exists some $\beta^{\prime} \in C$ such that $a b v=\beta^{\prime} b v$ which means $\left(a-\beta^{\prime}\right) b=0$ by a similar argument above. Using this in (4), we have

$$
\begin{aligned}
0 & =s\left(\left(x\left(a-\beta^{\prime}\right)+\left(c+\beta^{\prime}\right) x\right) b\right)^{n} \\
& =s\left(c+\beta^{\prime}\right)\left(x b\left(c+\beta^{\prime}\right)\right)^{n}
\end{aligned}
$$

for all $x \in R^{\prime}$. By Theorem 2 in 7, we have $s\left(c+\beta^{\prime}\right)=0$ or $b\left(c+\beta^{\prime}\right)=0$. Hence in either cases there exists some $\bar{\beta}^{\prime} \in C$ such that $a b=\beta^{\prime} b$.

Let $\left\{\mu_{0}, \mu_{1}, \ldots\right\}$ be a basis for $F$ over $C$ and let $\mu_{0}=1$. Writing $\beta^{\prime}=\beta \mu_{0}+\beta_{1} \mu_{1}+\ldots$ for $\beta, \beta_{1} \in C$, we obtain $\beta=\beta^{\prime}$ and $a b=\beta b$ for $\beta \in C$ that is $(a-\beta) b=0$. Thus it follows from the calculations in (3) that $s(c+\beta)=0$ or $b(c+\beta)=0$.

Proposition 4. Let $R$ be a prime ring and $s, a, b, c \in R$ with $s \neq 0, b \neq 0$. Suppose that $s(x a+c x b)^{n}=0$ for all $x \in R$, then there exists some $\beta \in C$ such that $a=\beta b$ and either $s(c+\beta)=0$ or $b(c+\beta)=0$.

Proof. It is well known that

$$
s(x a+c x b)^{n}=0
$$

holds for all $x \in Q_{m l}(R)$. First suppose that $R$ is not a generalized polynomial identity ring. Then the desired result follows from Lemma 2 .

Now suppose that $R$ is a GPI-ring. Since $Q_{m l}(R)$ is also a prime GPI-ring and a centrally closed prime ring then it follows from Martindale's Theorem in 13 that $Q_{m l}(R)$ is a primitive ring with nonzero socle $H$. Let $H$ be the socle of $Q_{m l}(R)$ and $H$ is a regular ring, that is for any $w \in H, w z w=w$ for some $z \in H$. Let $A n n_{r}(z)=\{x \in H \mid z x=0\}$ be the right annihilator of $z$ in $H$ and first consider the case $a, b \in H$. Let $w \in A n n_{r}(b)$. Substituting $x$ by $w x$ in (5) we have $s((w x) a+c(w x) b)^{n}=0$ and hence

$$
0=s((w x) a+c(w x) b)^{n} w=s w(x a w)^{n}
$$

for all $x \in Q_{m l}(R)$. Using Theorem 2 in 7 , we obtain either $s w=0$ or $a w=0$ that is $w \in A n n_{r}(s)$ or $w \in A n n_{r}(a)$. The first situation leads a contradiction so $w \in A n n_{r}(a)$ and hence $A n n_{r}(b) \subset A n n_{r}(a)$.

For $a, b \in H$ there exist $u, v \in H$ such that $a u a=a$ and $b v b=b$ since $H$ is a regular ring. Let $f=u a$ and $g=v b$ be two elements in $H$. It is easy to see that $f$ and $g$ are idempotents. Since $A n n_{r}(b) \subset A n n_{r}(a)$, then $A n n_{r}(g) \subset A n n_{r}(f)$ and 
also $(1-g) H \subseteq(1-f) H$. Thus we have $f(1-g)=0$. Here $a=a u a=a f=a f g=$ $a f v b \in H b$.

For general case let $w \in H$. Substituting $x$ by $x w$ in (5) we have

$$
0=s((x w) a+c(x w) b)^{n}
$$

for all $x \in Q_{m l}(R)$. For $w a, w b \in H$ by the calculations above it is clear that $w a \in H w b$,i.e., there exists some $t \in H$ such that $w a=t w b$. Writing this in (5), we have $s((x t+c x) w b)^{n}=0$. Using Lemma 3, there exists $\beta_{w} \in C$ such that $\left(t-\beta_{w}\right) w b=0$. Since $t w b=\beta_{w} w b$, then $w a=\beta_{w} w b$.

Fix an idempotent $e^{\prime} \in H$ such that $e^{\prime} a \neq 0$. Then

$$
e^{\prime} a=\beta e^{\prime} b
$$

for some $\beta \in C$. Let $f$ be an idempotent in $H$. Hence for some $\beta_{f} \in C$, we have

$$
f a=\beta_{f} f b .
$$

We wish to see that if $f a \neq 0$, then $\beta_{f}=\beta$. Indeed there exists an idempotent $h \in H$ such that $H e^{\prime}+H f=H h$ and

$$
h a=\beta_{h} h b .
$$

Here it is clear that $e^{\prime} h=e^{\prime}$ and $f h=h$.

$$
e^{\prime} a=e^{\prime} h a=e^{\prime} \beta_{h} h b=\beta_{h} e^{\prime} b .
$$

Using (6) in the last equation, we obtain $\beta e^{\prime} b=\beta_{h} e^{\prime} b$ and so $\beta=\beta_{h}$. Analogously

$$
f a=f h a=f \beta_{h} h b=\beta_{h} f b .
$$

In view of (7), we have $\beta_{h}=\beta_{f}$ and hence $\beta_{f}=\beta$. Until now we have seen that for an idempotent $f$ in $H$, there exists some $\beta \in C$ such that $f a=\beta f b$ in case $f a \neq 0$.

Now let $f \in H$ be an idempotent such that $f a=0$. Our aim is to see $f b=0$. By Litoff's Theorem (Theorem 4.3.11 in [1]), there exists an idempotent $h \in H$ such that $e^{\prime}, f \in h H h$. If $h a=0$, then $e^{\prime} a=e^{\prime} h a=0$, which is a contradiction. So we may assume that $h a \neq 0$. If $h a \neq 0$, then $(h-f) a \neq 0$. Here also note that $h-f$ is an idempotent. Since $h a \neq 0$ then by (8), $h a=\beta h b$ for some $\beta \in C$. Also $(h-f) a \neq 0$ implies $(h-f) a=\beta(h-f) b$ and so $f b=0$. Thus we obtain that if $f a=0$, then $f b=0$ for any idempotent $f \in H$.

In both cases, we have $f(a-\beta b)=0$ for all $f \in H$. Since $H$ is a regular ring then $H(a-\beta b)=0$ implies $a=\beta b$ for some $\beta \in C$. Using this result in (5), we have $s(x \beta b+c x b)^{n}=s(\beta+c)(x b(\beta+c))^{n}=0$ for all $x \in Q_{m l}$ which implies $s(c+\beta)=0$ or $b(c+\beta)=0$.

\section{The PRoof of the MAIN THEOREM}

Now we may state the proof of Theorem 1 . 
Proof. In view of Theorem 2.3 in [10], there exist a derivation $d: R \rightarrow Q_{m l}$ and $a \in Q_{m l}$ such that

$$
F(x)=x a+d(x) b
$$

for all $x \in R$. If $d$ is an $X$-inner derivation of $R$, then there exists some $c \in$ $Q_{m l}(R)$ such that $d(x)=[c, x]$ for all $x \in R$. Hence by the hypothesis, we have $s(x(a-c b)+c x b)^{n}=0$ for all $x \in R$. By Proposition 4 , there exists some $\beta \in C$ such that $a=(c+\beta) b$ and either $s(c+\beta)=0$ or $b(c+\beta)=0$.

Now consider the case when $d$ is not an $X$-inner derivation of $R$. Using Kharchenko's well known result in 9 , we have $s(x a+y b)^{n}=0$ for all $x, y \in R$. In particular $s(y b)^{n}=0$ for all $y \in R$. By Theorem 2 in $7, s=0$ or $b=0$ which leads a contradiction. So $d$ must be an $X$-inner derivation of $R$ and $F(x)=(c+\beta) x b$ for all $x \in R$ and either $s(c+\beta)=0$ or $b(c+\beta)=0$.

Example 5. Let $R$ be the ring of $2 \times 2$ upper triangular matrices over the field $\mathbb{F}$, i.e., $R=\left\{u e_{11}+w e_{12}+z e_{22} \mid u, w, z \in \mathbb{F}\right\}$. For the right b-generalized derivation $F$ of $R$, define $F(x)=x a+c x b$ for all $x \in R$. If we choose

$$
b=e_{11}+e_{12}+e_{22}, a=e_{12}, c=e_{11}, s=e_{22} \in R
$$

then, $s(F(x))^{n}=0$ for all $x \in R$ where $n \geq 1$ is a fixed positive integer but $s(c+\beta) \neq 0$ unless $\beta=0$ in $\mathbb{F}$.

\section{REFERENCES}

[1] Beidar, K. I., Martindale, W. S. III, Mikhalev, A. V., Rings with generalized identities, Marcel Dekker, Inc., New York, xiv+522 pp, 1996.

[2] Brešar, M., A note on derivations, Math. J. Okayama Univ., 32 (1990), 83-88.

[3] Chang, J.C., Annihilators of power values of a right generalized $(\alpha, \beta)$-derivation, Bull. Inst. Math. Acad. Sin., 4 (1) (2009), 67-73.

[4] Chuang, C. L., GPIs having coefficients in Utumi quotient rings, Proc. Amer. Math. Soc., 103 (3) (1988), 723-728.

[5] Erickson, T. S.; Martindale, W. S., 3rd; Osborn, J. M., Prime nonassociative algebras, Pacific J. Math., 60 (1975), No. 1, 49-63.

[6] Faith, C., Utumi, Y., On a new proof of Litoff's theorem, Acta Math. Acad. Sci. Hung., 14 (1967), 369-371.

[7] Felzenszwalb, B., On a result of Levitzki, Canad. Math. Bull. 21 (2) (1978), 241-242.

[8] Giambruno, A., Herstein, I.N., Derivations with nilpotent values, Rend. Circ. Mat. Palermo, 30 (2) (1981), 199-206.

[9] Kharchenko,V.K., Differential identities of semiprime rings, Algebra Logika, 18 (1979),86-119; Algebra Logic, (English translation), 18 (1979), 58-80.

[10] Kosan, M. T., Lee, T.K., b-generalized derivations having nilpotent values, J. Aust. Math. Soc., 96 (3) (2014), 326-337.

[11] Lee, T.K., Lin, J.S., A result on derivations, Proc. Amer. Math. Soc., 124 (1996), 1687-1691.

[12] Lee, T. K., Generalized derivations of left faithful rings, Comm. Algebra, 27(8) (1999), 40574073.

[13] Martindale, W. S. 3rd, Prime rings satisfying a generalized polynomial identity, J. Algebra, 12 (1969), 576-584. 